\title{
Fine tuning of the immune response by the Aryl Hydrocarbon Receptor
}

\author{
Caroline Pot • Francisco J. Quintana • Vijay K. Kuchroo
}

Received: 14 August 2013 / Accepted: 28 August 2013 / Published online: 9 October 2013

(C) Springer-Verlag Berlin Heidelberg 2013

This issue of Seminars in Immunopathology focuses on the role of the ligand-activated transcription factor aryl hydrocarbon receptor $(\mathrm{AhR})$ in controlling the immune response. $\mathrm{AhR}$ has been studied for many years by toxicologists as the receptor mediating the toxic effects of the environmental pollutant 2,3,7,8tetrachlorodibenzo-p-dioxin. However, AhR ligands are provided by the diet and are also generated in the gastrointestinal as a result of complex interactions between the host and the commensal flora. Moreover, endogenous ligands such the 2-(1'H-indole-3'carbonyl)-thiazole-4-carboxylic acid methyl ester or the tryptophan-derivative kynurenine are produced by the metabolism. Thus, the high degree of conservation of AhR throughout evolution and the existence of natural, nontoxic ligands suggests that AhR plays a physiological role in health and disease.

The past decade has witnessed significant advances in our understanding of the importance of $\mathrm{AhR}$ as a regulator of innate and adaptive immunity. This issue covers several aspects of the role of AhR in the control of the immune response and its relevance for the pathogenesis of immune related diseases. Lawrence and Vorderstrasse outline how AhR influences host response to viruses, bacteria, and parasites, noting how the effects of AhR signaling differ during the response to each pathogen and, therefore, suggesting the existence of pathogen-specific cues that modulate AhR activity. Quintana discusses in detail the role of AhR signaling in the control of T cell and dendritic cell differentiation, and its relevance for the regulation of central nervous system autoimmunity. Nguyen

\section{Pot}

Division of Neurology, Department of Pathology and Immunology, Geneva University Hospital, University of Geneva, Rue

Gabrielle-Perret-Gentil 4, 1211 Geneva 4, Switzerland

e-mail: Caroline.Pot@unige.ch

F. J. Quintana $・$ V. K. Kuchroo $(\bowtie)$

Center for Neurologic Diseases, Brigham and Women's Hospital,

Harvard Medical School, 77 Avenue Louis Pasteur, Boston, MA

02115, USA

e-mail: vkuchroo@rics.bwh.harvard.edu and Kishimoto evaluate the role of AhR during rheumatoid arthritis, and discuss new data on the control of microRNA expression by AhR. Subsequent contributions analyze the role of $\mathrm{AhR}$ as a master regulator of environmental interfaces such as the gut, the lungs, and the skin. Stange and Veldhoen describe the role of AhR in mucosal innate $\mathrm{T}$ cells and its importance in shaping the immune response to the microbiota. Qiu and Zhou discuss the role of AhR in controlling the function of innate lymphoid cells. Monteleone et al. evaluate the relevance of AhR signaling in gut inflammation, in particular during ulcerative colitis and Crohn's disease. The skin is another site that serves as a barrier to the external world, and AhR is implicated in skin homeostasis, as well as during infection and inflammation. Esser et al. examine in detail the regulation of skin immunity by AhR. Similarly, Beamer and Shepherd discuss the multiple roles of AhR in the development of lung diseases. Finally, Sherr and Monti analyze the intricate role of AhR during $B$ cell differentiation, the humoral response, and the development of $B$ cell malignancies.

In conclusion, $\mathrm{AhR}$ has important effects in the regulation of the immune response, making it a potential therapeutic target to control immune mediated disorders. We present this issue of Seminars in Immunopathology with the expectation that these reviews will bring us a step closer to understanding the complex roles of AhR in shaping immunity, and its exploitation as a therapeutic target. 